\title{
Cinética da frequência cardíaca nos domínios muito pesado e severo após manipulação dietética
}

\author{
Heart rate kinetics during very heavy and severe exercise \\ performed after dietary manipulation
}

\author{
Carlos Rafaell Correia de Oliveira ${ }^{1}$ \\ Flávio de Oliveira Pires 2,5 \\ Rômulo de Cássio Moraes Bertuzzi 3 \\ Fernando Roberto De-Oliveira ${ }^{4}$ \\ Maria Augusta Peduti Dal Molin Kiss 2 \\ Adriano Eduardo Lima-Silva ${ }^{1}$
}

\section{Universidade Federal de Alagoas. Faculdade de Nutrição. Grupo de Pesquisa em Ciências do Esporte. Maceió, AL. Brasil}

2 Universidade de São Paulo. Escola de Educação Física e Esporte. Laboratório de Determinantes Energéticos do Desempenho Esportivo. São Paulo, SP. Brasil.

3 Universidade de São Paulo. Escola de Educação Física e Esporte. Grupo de Estudos em Desempenho Aeróbio. São Paulo, SP. Brasil.

\section{Universidade Federal de} Lavras. Departamento de Educação Física. Núcleo de Estudos do Movimento Humano. Lavras, MG. Brasil.

5 Universidade Católica de Brasília - Brasília/DF, Brasil.

Recebido em 10/08/10 Revisado em 20/10/10 Aprovado em 26/11/10
Resumo - Pouco se conhece sobre a cinética da frequência cardíaca (FC) nos domínios muito pesado (MP) e severo (SE). O objetivo desse estudo foi identificar o ajuste que melhor descreveria a cinética da FC nesses domínios e comparar os parâmetros derivados desses ajustes entre uma situação de alto $(\mathrm{ACHO})$, baixo $(\mathrm{BCHO})$ e normal $(\mathrm{C})$ disponibilidade de carboidrato. Doze homens realizaram três testes até a exaustão em um dos domínios após dois dias de dieta com $\mathrm{ACHO}, \mathrm{BCHO}$ ou C. A intensidade de MP foi $\triangle \mathrm{LW} 75 \%$ (75\% da diferença entre o $\mathrm{VO}_{2 \max }$ e LL2) e $\mathrm{SE} 115 \%$ do $\mathrm{VO}_{2 \max }$, identificadas em um teste incremental prévio (20W/3min). A FC foi ajustada pelas equações mono-exponencial (Mono) e bi-exponencial (Bi). No domínio MP, a média do somatório de resíduos do Bi foi significativamente menor do que mono $(\mathrm{P}<0,05)$. No domínio $\mathrm{SE}$, a média do somatório de resíduos entre os modelos não foi significativamente diferente $(p>0,05)$. No domínio MP, os parâmetros do ajuste Bi não apresentaram diferenças significantes $(\mathrm{p}>0,05)$ entre as situações $\mathrm{C}, \mathrm{ACHO}$ e $\mathrm{BCHO}$, respectivamente. No domínio $\mathrm{SE}$, os parâmetros do ajuste Mono não diferiram entre as situações $(p>0,05)$. Contudo, a constante de tempo foi significativamente reduzida na situação de BCHO quando comparado a $\mathrm{ACHO}(51,5 \pm$ $26,4 \mathrm{~s}$ vs $65,4 \pm 34,1 \mathrm{~s} ; \mathrm{p}<0,05)$. Portanto, os ajustes $\mathrm{Bi}$ e Mono parecem representar melhor a cinética da FC nos domínios MP e SE, respectivamente. Além disso, a disponibilidade de $\mathrm{CHO}$ parece ter interferência apenas na resposta da FC em exercício de domínio SE. Palavras-chave: Modelos matemáticos; Frequência cardíaca; Carboidrato; Esforço físico.

Abstract - Little is known about heart rate (HR) kinetics during exercise in the very heavy (VH) and severe (SE) intensity domains. The objective of this study was to describe mathematically the HR kinetics during exercise performed in these intensity domains and to compare the parameters derived from these models between situations of high ( $\mathrm{HCHO})$, low $(\mathrm{LCHO})$ and control $(\mathrm{C})$ carbohydrate availability. Twelve men performed three trials to exhaustion in the VH or SE domains after diet manipulation with $\mathrm{HCHO}, \mathrm{LCHO}$ and $\mathrm{C}$. The VH intensity was $\triangle L W 75 \%$ (75\% of the difference between $\mathrm{VO}_{2 \max }$ and LL2) and SE was $115 \%$ of $\mathrm{VO}_{2 \max }$ identified in a previous incremental test $(20 \mathrm{~W} / 3 \mathrm{~min})$. HR responses were mathematically fitted by mono- and biexponential functions. In the VH domain, the residual sum of squares (RSS) obtained with the biexponential model was significantly lower than that obtained with the monoexponential model $(P<0.05)$. In the SE domain, no significant difference in RSS was observed between the mathematical models $(P>0.05)$. In the VH domain, there were no significant differences in biexponential parameters between the $\mathrm{HCHO}, \mathrm{LCHO}$ and $\mathrm{C}$ conditions. In the SE domain, there were no significant differences in monoexponential parameters between the $\mathrm{HCHO}, \mathrm{LCHO}$ and $\mathrm{C}$ conditions, although the time constant of the monoexponential model was significantly reduced in $\mathrm{LCHO}$ when compared to $\mathrm{HCHO}(51.5 \pm 26.4$ vs $65.4 \pm 34.1 \mathrm{~s} ; \mathrm{P}<0.05)$. The bi-and monoexponential mathematical models seem to be the best description of $\mathrm{HR}$ responses during exercise performed in the HV and SE intensity domains, respectively. In addition, carbohydrate availability only seems to affect HR kinetics during exercise performed at SE intensity. Key words: Mathematical models; Heart rate; Carbohydrate; Physical exertion. 


\section{INTRODUÇÃO}

Os domínios de intensidade podem ser divididos em moderado $(\mathrm{MO})$, pesado $(\mathrm{PE})$, muito pesado (MP) e severo $(\mathrm{SE})^{1}$. O comportamento de algumas variáveis fisiológicas durante a execução de exercícios de cargas constantes parece responder em função do domínio metabólico em que se está realizando o esforço. Entre as diversas variáveis fisiológicas estudadas, uma é a resposta do consumo de oxigênio $\left(\mathrm{VO}_{2}\right)^{1-3}$. Na literatura ${ }^{1,3}$, é bem documentado que o modelo monoexponencial (Mono) caracteriza a cinética do $\mathrm{VO}_{2}$ para os domínios de esforço $\mathrm{MO}$ e $\mathrm{SE}$, enquanto o modelo biexponencial (Bi) descreve o comportamento nos domínios PE e MP. Acredita-se que a segunda exponencial nos domínios PE e MP seja decorrente do componente lento (CL) do $\mathrm{VO}_{2}, 5$. Entretanto, apesar desse bem caracterizado modelo de resposta para o $\mathrm{VO}_{2}$, muito menos é conhecido sobre a cinética de outras variáveis fisiológicas, como por exemplo, a frequência cardíaca (FC). Um modelo similar de resposta poderia ser esperado para essas duas variáveis, uma vez que $\mathrm{o} \mathrm{VO}_{2}$ durante o exercício, está relacionado ao trabalho cardíaco realizado.

Alguns estudos ${ }^{6-8}$ ressaltaram que quando o exercício é realizado no domínio MO, a FC apresenta um comportamento Mono, ao passo que, no domínio PE, a cinética da FC apresenta um comportamento Bi. Possivelmente, este aumento adicional na FC no domínio PE seja resultante do desequilíbrio entre a demanda energética imposta pelos músculos ativos e a capacidade do sistema cardiovascular em suprir essa demanda? Além disso, com o passar do tempo, é possível se detectar uma elevação do ritmo cardíaco devido a um fenômeno conhecido como "drift" cardíaco, no qual há uma diminuição gradual no volume sistólico e uma elevação na FC causada por um aumento na temperatura e pela desidratação, ${ }^{910}$. Apesar dessa descrição cinética para a resposta da FC nos domínios $\mathrm{MO}$ e $\mathrm{PE}$, pouco se conhece sobre a resposta dessa variável quando o exercício é realizado em intensidades próximas ao consumo de oxigênio máximo $\left(\mathrm{VO}_{2 \max }\right)$, como nos domínios $\mathrm{MP}$ e SE.

Além da intensidade do exercício, outros fatores também parecem afetar o comportamento da FC durante o exercício de carga constante. Alguns estudos $^{11,12}$ demonstraram que a manipulação dos estoques de carboidrato $(\mathrm{CHO})$ pode afetar a atividade do sistema nervoso autônomo. Sasaki et al. ${ }^{11}$ observaram um aumento dos níveis de norepinefrina plasmática durante o exercício a 70\% do
$\mathrm{VO}_{2 \text { máx }}$, após sete dias de dieta com baixo conteúdo de $\mathrm{CHO}$, quando comparado com uma dieta com alto conteúdo de CHO. Da mesma forma, Helge et al..$^{12}$ demonstraram maiores níveis plasmáticos de norepinefrina e FC durante o exercício a $80 \%$ do $\mathrm{VO}_{2 \text { máx }}$, após uma dieta pobre em $\mathrm{CHO}$ durante 7 dias. Eles sugeriram que o aumento na atividade simpática foi o resultado da baixa disponibilidade de $\mathrm{CHO}$. Recentemente, foi sugerido que atletas que utilizam dietas com baixo teor de $\mathrm{CHO}$, visando um aumento no desempenho em decorrência do aumento na oxidação de gordura e redução na oxidação de glicogênio muscular (GM), podem apresentar um maior estresse cardiovascular, acarretando um aumento na atividade simpática e na $\mathrm{FC}^{13,14}$. Consequentemente, a baixa disponibilidade de $\mathrm{CHO}$ poderia provocar alterações no comportamento cinético da FC. Na literatura, está bem documentado que uma baixa disponibilidade de $\mathrm{CHO}$ poderia alterar os parâmetros cinéticos do $\mathrm{VO}_{2}$, principalmente, em exercícios de alta intensidade $^{15-17}$, mas não é conhecido se o mesmo ocorre para os parâmetros cinéticos da FC. Assumindo que existe uma relação entre a cinética do $\mathrm{VO}_{2}$ e da $\mathrm{FC}$, parece razoável imaginar que a disponibilidade de $\mathrm{CHO}$ poderia alterar a cinética desse último. No entanto, ainda não é conhecido o impacto da manipulação de $\mathrm{CHO}$ sobre os parâmetros derivados dos ajustes Mono e Bi da resposta cinética da FC, principalmente, nos domínios MP e SE. Além disso, a interação entre a manipulação de $\mathrm{CHO}$ e a intensidade do exercício sobre a cinética da FC ainda precisa ser estabelecida.

Desta forma, o objetivo desse estudo foi identificar o ajuste que melhor descreveria a cinética da FC em exercício nos domínios MP e SE, além de comparar os parâmetros derivados desses ajustes entre situações com diferente disponibilidade de $\mathrm{CHO}$ dietético. Levando-se em consideração que os modelos Bi e Mono descrevem melhor a cinética do $\mathrm{VO}_{2}$ nas intensidades MP e SE, respectivamente, a primeira hipótese deste estudo foi que esses ajustes descreveriam melhor, também, a cinética da FC nesses domínios. Além disso, a segunda hipótese é que a disponibilidade de $\mathrm{CHO}$ dietético poderia alterar os parâmetros derivados do melhor ajuste exponencial.

\section{PROCEDIMENTOS METODOLÓGICOS}

\section{Sujeitos}

Doze homens saudáveis $(28,5 \pm 7$ anos; $74,8 \pm 9 \mathrm{~kg}$; $178,6 \pm 4,4 \mathrm{~cm} ; 12,9 \pm 3,2 \% ; 46,9 \pm 9,7 \mathrm{ml} \cdot \mathrm{kg}^{-1} \cdot \mathrm{min}$ 
$\left.{ }^{1}\right)$ participaram do estudo. Cada um dos participantes foi informado sobre os riscos e procedimentos do estudo e assinaram um termo de consentimento, concordando em participar voluntariamente do experimento. Os procedimentos adotados no presente estudo foram aprovados pelo Comitê de Ética para estudos com humanos da Escola de Educação Física e Esporte da Universidade de São Paulo.

\section{Desenho experimental}

Na primeira visita, foi realizada uma avaliação antropométrica para determinação da massa corporal, estatura e percentual de gordura, este último utilizando as dobras cutâneas do peitoral, abdômen e coxa ${ }^{18}$. Logo em seguida, os indivíduos realizaram um teste progressivo no cicloergômetro eletromagnético (Ergo Fit 167, Pirmansens, Germany) para determinação do $\mathrm{VO}_{2 \max }$ e do primeiro (LL1) e segundo (LL2) limiares de lactato. Na segunda visita, um teste controle (C) foi realizado no domínio MP $(\mathrm{n}=6)$ ou SE $(\mathrm{n}=6)$, nas intensidades $\triangle \mathrm{LW} 75 \%$ e $115 \% \mathrm{VO}_{2 \max }$, respectivamente. A intensidade de $\triangle \mathrm{LW} 75 \%$ corresponde a $75 \%$ da diferença entre a carga máxima e o LL2, ao passo que intensidade de $115 \% \mathrm{VO}_{2 \max }$ corresponde a $115 \%$ do consumo máximo de oxigênio. Para a determinação da carga de trabalho no domínio MP, foi escolhido como referência os limiares de lactato ao invés de exclusivamente o $\mathrm{VO}_{2 \max }$, pois o primeiro tem mostrado um nível mais consistente de estresse metabólico entre diferentes indivíduos ${ }^{15,19}$. Nas visitas três e quatro, foi realizado um teste experimental na mesma carga do teste controle, após 48 horas de manipulação dietética com baixo (BCHO) ou alto (ACHO) conteúdo de $\mathrm{CHO}$. A ordem de aplicação das dietas foi determinada de forma contrabalançada com uma semana de intervalo entre elas.

\section{Teste progressivo}

Os indivíduos iniciaram o teste progressivo pedalando durante cinco minutos à $50 \mathrm{~W}$ para aquecimento. Posteriormente, foram acrescentados $20 \mathrm{~W}$ a cada três minutos, até a exaustão voluntária. Durante todo o teste, os indivíduos mantiveram a frequência de rotação do pedal entre 60 e 70 rev. $\min ^{-1}$. O teste foi interrompido quando os indivíduos não conseguiram manter a rotação dentro desse intervalo.

$\mathrm{O} \mathrm{VO}_{2}, \mathrm{VCO}_{2}$ e $\mathrm{VE}$ foram mensurados respiração a respiração durante todo o teste (Quark $b^{2}$; Cosmed, Itália). Os seguintes critérios foram utilizados para verificar se os indivíduos atingiram o $\mathrm{VO}_{2 \max }: 1$ ) aumento inferior a $150 \mathrm{ml} \cdot \mathrm{min}^{-1}$ no $\mathrm{VO}_{2}$ após o incremento da carga; 2) razão de trocas respiratórias $(\mathrm{R}) \geq 1,10 ; 2$ ) $\mathrm{FC} \geq$ a $90 \%$ da máxima predita pela idade. Vinte e cinco microlitros de sangue foram retirados do lóbulo da orelha ao final de cada estágio para posterior análise da concentração de lactato sanguíneo [La] (analisador automático YSI 1500 Sport, Yellow Springs Instrumento, Yellow Springs, OH). As [La] foram plotadas em função da potência e o $\mathrm{LL}_{1}$ e o $\mathrm{LL}_{2}$ foram identificados a partir de um ajuste de três segmentos de reta na curva lactato versus intensidade ${ }^{20}$.

\section{Teste controle}

Os indivíduos realizaram um teste controle no período da manhã, com o indivíduo em jejum de 8-12 horas e sem manipulação de dieta. Após cinco minutos de aquecimento em 50W, a carga foi ajustada para a intensidade estipulada (MP ou SE). O teste foi realizado até a exaustão. A FC foi mensurada durante todo o teste (Polar S810i, Finlândia).

\section{Controle dietético}

Quarenta e oito horas antes dos testes experimentais, um protocolo de exercício foi realizado para reduzir as reservas endógenas de $\mathrm{CHO}$, principalmente, as de GM. Os indivíduos pedalaram durante 90 minutos a 50\% da diferença entre o LL1 e o LL2, seguido por seis séries a $125 \%$ do $\mathrm{VO}_{2 \max }$, intercalados por um minuto de repouso. Esse protocolo de depleção de GM foi previamente validado por Gollnick et al. ${ }^{21}$ que demonstraram uma diminuição do conteúdo de GM de ambos os tipos de fibra (tipo I e II). Além disso, Gollnick et al. ${ }^{21}$ demonstraram que, quando um exercício intermitente intenso é realizado até a exaustão, o GM de ambos os tipos de fibras é depletado, independentemente da intensidade usada (120-150\% da potência aeróbica máxima). Dessa forma, é muito provável que a reserva de GM tenha sido depletada com o protocolo usado no presente estudo.

Nas 48 horas seguintes, os indivíduos realizaram uma dieta isocalórica com $\mathrm{BCHO}(10 \%$ de carboidrato, $35 \%$ de gordura e $55 \%$ de proteína) ou $\mathrm{ACHO}$ (80\% de carboidrato, 10\% de gordura e 10\% de proteína) até o dia do teste experimental. Durante os dois dias de realização das dietas, os indivíduos foram orientados a registrarem em forma de recordatório, todos os alimentos consumidos nesse período, para posterior comparação com o que foi prescrito. A validade desse tipo de dieta para manter baixas as reservas de $\mathrm{GM}(\mathrm{BCHO})$ ou aumentá-las $(\mathrm{ACHO})$ foi descrita anteriormente $\mathrm{e}^{13}$. 


\section{Testes experimentais}

Os indivíduos compareceram ao laboratório no período da manhã e realizaram o mesmo teste aplicado na situação controle, com os mesmos procedimentos e medidas. Um período de sete dias foi adotado para wash-out entre as duas situações experimentais ${ }^{22}$.

\section{Análise dos dados}

Os dados de FC foram transferidos para um "software" (Origin 6.0), onde foram convertidos a cada 1 s e em seguida, ajustados por duas equações exponenciais (figura 1):

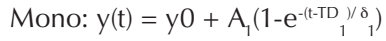

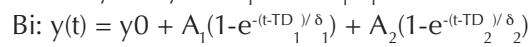

Onde: y(t) é a FC em um determinado tempo; y0 é a FC de base; $A_{1}$ e $A_{2}$ correspondem à amplitude da primeira e segunda exponenciais, respectivamente; TD e TD referem-se ao tempo de atraso da primeira e segunda exponenciais, respectivamente $\delta_{1}$ e $\delta_{2}$ referem-se às constantes de tempo da primeira e segunda exponenciais, respectivamente.
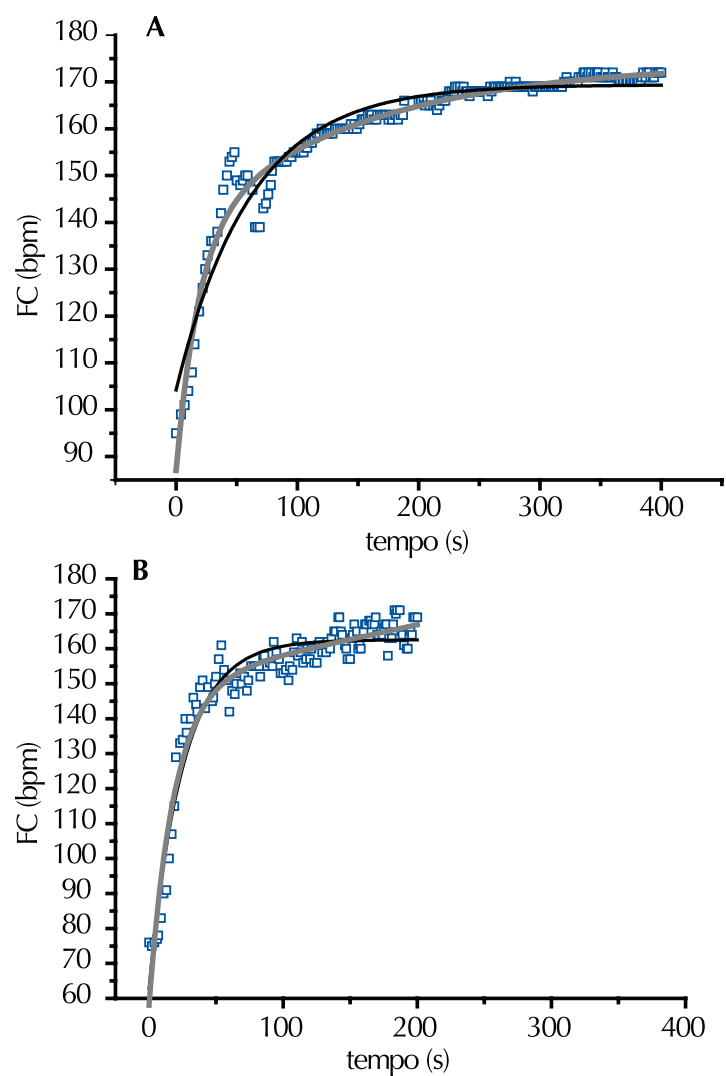

Figura 1. Cinética da frequência cardíaca durante o exercício muito pesado (painel A) e severo (painel B) com os ajustes mono (linha preta) e biexponencial (linha cinza) em dois indivíduos representativos da amostra.
A somatória dos resíduos ao quadrado (SRQ) e o $R^{2}$ de cada equação foram comparados, utilizando o teste $t$ de Student para amostras dependentes. $\mathrm{O}$ modelo mais simples (Mono) foi considerado como o mais adequado quando não houve diferenças significantes, baseado no princípio da Parcimônia.

A escolha do melhor ajuste que representaria a cinética da FC em cada domínio (MP e SE) foi decidida no teste controle. Em seguida, os parâmetros derivados do melhor ajuste para cada um dos domínios foram comparados entre as três situações de diferentes disponibilidades de $\mathrm{CHO}$ dietético, utilizando ANOVA de um caminho e teste post-hoc de Bonferroni. Todos os procedimentos estatísticos foram realizados no "software" SPSS (versão 13.0), adotando um nível de significância de 5\% (p < 0,05).

\section{RESULTADOS}

O tempo de exaustão para o domínio MP foi menor na condição $\mathrm{BCHO}$ do que em $\mathrm{ACHO}$, mas ambos não foram diferentes do controle (C: 9,54 $\pm 4,12$ min; ACHO: $10,72 \pm 5,08$ min e; $\mathrm{BCHO}: 8,12 \pm$ 2,49 min). Para o domínio SE, o tempo de exaustão foi menor na situação de $\mathrm{BCHO}$ do que em $\mathrm{ACHO}$ e C (C: 3,59 $\pm 0,72 \mathrm{~min} ; \mathrm{ACHO}: 4,26 \pm 0,69 \mathrm{~min}$ e; $\mathrm{BCHO}: 2,91 \pm 0,56 \mathrm{~min})$.

\section{Comportamento da FC no MP}

A SRQ foi significativamente maior para o ajuste Mono $(6730,4 \pm 5221)$ do que para o ajuste $\mathrm{Bi}(2425,4$ $\pm 1367,1 ; \mathrm{P}<0,05)$. Da mesma maneira, os valores de $\mathrm{R}^{2}$ foram menores para o ajuste Mono $(0,956$ $\pm 0,021)$ do que para o ajuste $\mathrm{Bi}(0,981 \pm 0,011$; $\mathrm{p}<0,05)$. Assim, o modelo Bi foi considerado como o mais adequado para descrever a cinética da FC.

\section{Comportamento da FC no SE}

Nesse domínio, o Mono foi considerado como o melhor ajuste, uma vez que o SRQ entre os modelos não foi significativamente diferente (Mono: 1727,9 $\pm 2106,9$ vs $\mathrm{Bi}: 1335,4 \pm 1723 ; \mathrm{p}>0,05)$ e nem os valores de $\mathrm{R}^{2}$ (Mono: 0,980 $\pm 0,019$ vs Bi: 0,984 $\pm 0,016, p>0,05$ ).

\section{Comparação dos parâmetros entre as situações de $\mathrm{CHO}$}

No domínio MP, os parâmetros derivados do ajuste Bi e o $R^{2}$ não apresentaram diferenças significantes $(p>0,05)$ entre as situações (tabela 1). O mesmo aconteceu no domínio SE, com exceção da $\delta_{1}$, que foi significativamente menor na dieta $\mathrm{BCHO}$ do que na dieta $\mathrm{ACHO}$ (tabela 2). 
Tabela 1. Parâmetros do ajuste biexponencial no domínio Muito Pesado nas situações controle (C), alto (ACHO) e baixo (BCHO) conteúdo de carboidrato dietético.

\begin{tabular}{lcccc}
\hline Parâmetros & C & ACHO & BCHO & Valor de P \\
\hline$y 0$ & $75,6 \pm 1,1$ & $76,1 \pm 2,5$ & $76,3 \pm 2,2$ & 0,69 \\
$A_{1}$ & $83,5 \pm 0,6$ & $84 \pm 3,6$ & $84,4 \pm 3$ & 0,18 \\
$\delta_{1}$ & $20,7 \pm 6,5$ & $34,4 \pm 18,5$ & $20,1 \pm 9,2$ & 0,99 \\
$A_{2}$ & $29,7 \pm 14$ & $64,4 \pm 63,1$ & $30,3 \pm 12,5$ & 0,69 \\
$\delta_{2}$ & $305,9 \pm 119$ & $852,8 \pm 891,3$ & $320,1 \pm 190,8$ & 0,09 \\
$R^{2}$ & $0,982 \pm 0,011$ & $0,976 \pm 0,033$ & $0,991 \pm 0,003$ & 0,95 \\
\hline
\end{tabular}

Tabela 2. Parâmetros do ajuste monoexponencial no domínio Severo nas situações controle $(\mathrm{C})$, alto $(\mathrm{ACHO})$ e baixo $(\mathrm{BCHO})$ conteúdo de carboidrato dietético.

\begin{tabular}{lcccc}
\hline Parâmetros & C & ACHO & BCHO & Valor de P \\
\hline$y 0$ & $80,1 \pm 5$ & $81 \pm 6,1$ & $79,9 \pm 5,4$ & 0,18 \\
$A_{1}$ & $90,1 \pm 9,7$ & $91,5 \pm 4,9$ & $89 \pm 6,6$ & 0,36 \\
$\delta_{1}$ & $58,7 \pm 33,8$ & $65,4 \pm 34,1$ & $51,5 \pm 26,4$ & $0,03 *$ \\
$R^{2}$ & $0,978 \pm 0,021$ & $0,966 \pm 0,026$ & $0,981 \pm 0,012$ & \\
\hline
\end{tabular}

* Diferenças significantes entre ACHO e BCHO $(p<0,05)$.

\section{DISCUSSÃO}

Os principais achados deste estudo foram que nos domínios MP e SE os ajustes que melhor descreveram a cinética da $\mathrm{FC}$ foram o Bi e o Mono, respectivamente. Além disso, as diferentes situações de $\mathrm{CHO}$ dietético $(\mathrm{C}, \mathrm{ACHO}$ e $\mathrm{BCHO})$ não tiveram influência sobre os parâmetros derivados do modelo Bi no domínio de intensidade MP. Entretanto, no domínio $\mathrm{SE}$, a $\delta_{1}$ foi reduzida com a dieta $\mathrm{BCHO}$.

No domínio MP, o modelo Bi gerou menores valores de $\mathrm{SR}$ e maiores de $\mathrm{R}^{2}$, o que sugere que esse ajuste é o mais adequado para esse domínio. Assumindo uma associação entre $\mathrm{o} \mathrm{VO}_{2}$ e $\mathrm{FC}$, este resultado corrobora os resultados obtidos por Ozyener et al. ${ }^{1}$, nos quais o modelo $\mathrm{Bi}$ representou melhor a cinética do $\mathrm{VO}_{2}$ nesse domínio. Além disso, levando-se em consideração que no presente estudo, assim como no de Ozyener et al. ${ }^{1}$, não foi alcançado um estado de equilíbrio durante o exercício, sugere-se que um CL é evidente tanto para o $\mathrm{VO}_{2}$ quanto para a $\mathrm{FC}$ quando o esforço é realizado no domínio MP. A presença de um CL para o domínio MP pode ser justificada pelo fato de que o consumo de oxigênio pode aumentar até atingir o $\mathrm{VO}_{2 \text { máx }} 4,23$. Portanto, como o $\mathrm{VO}_{2}$ é diretamente proporcional ao débito cardíaco que, por sua vez, é o produto entre o volume sistólico e a FC, parece sensato esperar que a resposta da FC acompanhe a do $\mathrm{VO}_{2}$, como demonstrado pelo melhor ajuste $\mathrm{Bi}$.

No domínio SE, o ajuste Mono foi considerado o mais adequado. Ozyener et al. ${ }^{1}$ destacaram que o ajuste Mono descreve melhor a curva do $\mathrm{VO}_{2}$ no domínio SE, sugerindo que não há presença de CL do $\mathrm{VO}_{2}$. No presente estudo, o início do CL do domínio MP foi evidente a partir de $\sim 3,9$ minutos, enquanto o tempo de exaustão no domínio SE foi de apenas $\sim 3,5$ minutos. Desse modo, pode ser que no domínio SE não tenha dado tempo para a manifestação do CL. Uma consideração semelhante foi observada por Ozyener et al. ${ }^{1}$, onde no domínio MP o CL do $\mathrm{VO}_{2}$ começou a surgir em 137 segundos e o tempo de duração do teste no SE foi de 150 segundos. Portanto, no domínio SE, devido à intensidade e duração, torna-se improvável a existência de uma segunda exponencial.

Os parâmetros derivados do ajuste Bi no domínio MP não foram diferentes entre as situações de $\mathrm{CHO}$. Alguns estudos ${ }^{11,12,14,15}$ demonstraram que a redução na disponibilidade de $\mathrm{CHO}$ pode aumentar a atividade simpática. A literatura ${ }^{14,24,25}$ aponta que, com o aumento da intensidade, existe uma retirada vagal, com uma redução significativa da variabilidade da FC (VFC) e um aumento da FC devido à ativação simpática. Em exercícios próximos ao $\mathrm{VO}_{2 \text { máx }}$ a $\mathrm{VFC}$ tende a atingir valores próximos a zero, sugerindo uma atividade simpática máxi$\mathrm{ma}^{26,27}$. Estes resultados talvez indiquem que a dieta $\mathrm{BCHO}$ não tenha efeito aditivo sobre o sistema simpático quando medido a esse nível de atividade, sugerindo que nesse domínio a ativação simpática é influenciada muito mais pela alta intensidade do exercício do que pela manipulação dietética.

No domínio SE, o parâmetro $A_{1}$ não diferenciou entres as situações, mas a $\delta_{1}$ foi menor na 
dieta $\mathrm{BCHO}$. $\mathrm{O}$ fato dos valores do parâmetro $\mathrm{A}_{1}$ terem sido similares entre as condições dietéticas, indicam que a dieta $\mathrm{BCHO}$ não influencia o valor final atingido de FC, da mesma maneira como demonstrado para o domínio MP. Como encontrado em outros estudos ${ }^{11,12,14,15}$, a baixa disponibilidade de $\mathrm{CHO}$ aumenta a atividade simpática, mas ao final do esforço de alta intensidade a atividade simpática é máxima e a intensidade talvez tenha mais efeito sobre a regulação da FC do que a dieta. Dessa forma, como SE é uma intensidade maior do que MP, parece razoável imaginar que realmente exista um nível de ativação máxima do sistema simpático nessa intensidade. Contudo, a redução da $\delta_{1}$ sugere que a manipulação dietética antecipa a resposta da FC, mas não é possível a partir desses dados inferir se isso é devido a uma ativação simpática precoce ou uma retirada tardia da atividade parassimpática. Entretanto, baseado nos achados que sugerem um aumento da atividade simpática com a baixa disponibilidade de $\mathrm{CHO}^{11,12,14,15}$, parece razoável imaginar que talvez tenha tido uma ativação simpática precoce para atender a maior demanda energética imposta pelo sistema músculo-esquelético, decorrente da alta intensidade do esforço e da baixa disponibilidade de $\mathrm{CHO}$ intramuscular?

O presente estudo apresenta algumas limitações. Primeiramente, um número relativamente baixo de sujeitos foi utilizado, o que pode ter influenciado algumas das conclusões do estudo. Contudo, o baixo número de sujeitos é decorrente da complexidade do desenho experimental, com muitas medidas e dias de testes. Da mesma maneira, um melhor ajuste poderia ser encontrado caso os dados analisados tivessem sido gerados por média de valores obtidos em mais de um teste para cada situação. Entretanto, devido ao número elevado de manipulações para atender essa proposta, torna-se inviável do ponto de vista experimental tal aplicação. Além disso, poucos estudos investigaram o ajuste cinético da FC nesses domínios, prejudicando a comparação dos nossos resultados com os da literatura. Outra limitação diz respeito à precisão dos parâmetros cinéticos da FC com apenas uma transição, especialmente, quando há manipulação experimental. Contudo, a aquisição de dados da transição da FC do repouso para o exercício apresenta elevada reprodutibilidade ${ }^{28}$. Além disso, a utilização de apenas uma transição é comum devido à limitação experimental inerente de estudos com múltiplas mensurações e manipulações ${ }^{28,29}$. Caso optássemos em realizar três testes em cada condição experimental para obter maior precisão dos parâmetros, seriam necessárias mais 15 visitas ao laboratório, tornando inviável a execução de qualquer estudo experimental desta natureza, conforme documentado em estudos recentes ${ }^{13,14,30}$. Dessa forma, a utilização de médias a cada um ou cinco segundos parece ser suficiente para analisar pontos de dados com características similares a curva FC-tempo e obter uma boa estimativa dos parâmetros estudados no presente estudo ${ }^{3}$. Assim, optou-se em realizar apenas uma condição experimental para cada situação, sendo pouco provável que isso possa ter afetado significativamente os nossos resultados.

Em conclusão, os ajustes Bi e Mono parecem representar melhor a cinética da FC nos domínios MP e SE, respectivamente. A manipulação de $\mathrm{CHO}$ dietético não parece interferir na resposta da FC no domínio MP, mas parece acelerar o seu tempo de resposta no domínio SE. Assim, dietas com baixo conteúdo de $\mathrm{CHO}$, comumente utilizadas pelos atletas na expectativa de aumentar o desempenho devido ao aumento na oxidação de gordura e redução na oxidação de GM, podem acelerar a ativação simpática em exercício supramáximo, como o domínio SE, gerando, possivelmente, um maior estresse cardiovascular ao atleta.

\section{REFERÊNCIAS BIBLIOGRÁFICAS}

1. Ozyener F, Rossiter HB, Ward SA, Whipp BJ. Influence of exercise intensity on the on and off transient kinetics of pulmonary oxygen uptake in humans. J Physiol 2001;533(3): 891-902.

2. Lima-Silva AE, De Oliveira FR. Consumo de oxigênio durante o exercício físico: aspectos temporais e ajustes de curvas. Rev Bras Cineantropom Desempenho Hum 2004;6(2):73-82.

3. Barstow TJ, Molé PA. Linear and nonlinear characteristics of oxygen uptake kinetics during heavy exercise. J Appl Physiol 1991;71:2099-106.

4. Gaesser GA, Poole DC. The slow component of oxygen uptake kinetics in humans. Exer Sport Sci Rev 1996;24:35-71.

5. Borrani F, Candau R, Millet GY, Perrey S, Fuchslocher J, Rouillon JD. Is the VO2 slow component dependent on progressive recruitment of fast-twitch fibers in trained runners? J Appl Physiol 2001;90(6):2212-20.

6. Bearden S, Moffatt RJ. VO2 and heart rate kinetics in cycling: transitions from an elevated baseline. J Appl Physiol 2001;90:2081-7.

7. Engelen M, Porszasz J, Riley M, Wasserman K, Maehara $\mathrm{K}$, Barstow TJ. Effects of hypoxic hypoxia on $\mathrm{O} 2$ uptake and heart rate kinetics during heavy exercise. J Appl Physiol 1996;81(6):2500-8.

8. Rumenig E, Bertuzzi RCM, Nakamura FY, Franchini E, Matsushigue KA, Kiss MAPDM. Cinética e variabilidade da freqüência cardíaca mediante exercício físico predominantemente aeróbio: influência da intensidade e do tempo de análise. Rev Bras de Educ Fis 2007;21(3):205-18. 
9. Dawson EA, Shave R, George K, Whyte G, Ball D, Gaze D, et al. Cardiac drift during prolonged exercise with echocardiographic evidence of reduced diastolic function of the heart. Eur J Appl Physiol 2005;94:305-9.

10. Achten J, Jeukendrup AE. Heart rate monitoring: applications and limitations. Sports Med 2003;30(7):517-38.

11. Sasaki H, Hotta N, Ishiko T. Comparison of sympatho-adrenal activity during endurance exercise performed under high- and low-carbohydrate diet conditions. J Sports Med Phys Fitness 1991;31:407-12.

12. Helge JW, Richter EA, Kiens B. Interaction of training and diet on metabolism and endurance during exercise in man. J Physiol 1996;492(Pt 1):293-306.

13. Baldwin J, Snow RJ, Gibala MJ, Garnham A, Howarth K, Febbraio MA. Glycogen availability does not affect the TCA cycle or TAN pools during prolonged, fatiguing exercise. J Appl Physiol 2003;94:2181-7.

14. Lima-Silva AE, Bertuzzi RCM, Pires FO, Fronchetti L, Gevaerd MS, De-Oliveira FR. A low carbohydrate diet affects autonomic modulation during heavy but not moderate exercise. Eur J Appl Physiol 2010;108:1133-40.

15. Lima-Silva AE, Pires FO, Bertuzzi RC, Lira FS, Casarini D, Kiss MA. Low carbohydrate diet affects the oxygen uptake on-kinetics and rating of perceived exertion in high intensity exercise. Psychophysiology 2010. DOI: 10.1111/j.1469-8986.2010.01059.x

16. Carter H, Pringle JS, Boobis L, Jones AM, Doust JH. Muscle glycogen depletion alters oxygen uptake kinetics during heavy exercise. Med Sci Sports Exerc 2004;36:965-72.

17. Osborne MA, Schneider DA. Muscle glycogen reduction in man: relationship between surface EMG activity and oxygen uptake kinetics during heavy exercise. Exp Physiol 2006; 91:179-89.

18. Jackson AS, Pollock ML. Generalized equations for predicting body density of men. Brit J Nutr 1978;40:497-504.

19. Lira FS, Zanchi NE, Lima-Silva AE, Pires FO, Bertuzzi RC, Santos RV, Caperuto EC, Kiss MA, Seelaender M. Acute high-intensity exercise with low energy expenditure reduced LDL-c and total cholesterol in men. Eur J Appl Physiol 2009;107(2):203-10.

20. Ribeiro JP, Yang J, Adams RP, Kuca B, Knutten HG. Effect of different incremental exercise protocols on the determination of lactate and ventilatory thresholds. Braz J Med Biol Res 1986;19:109-17.

21. Gollnick PD, Piehl K, Saltin B. Selective glycogen depletion pattern in human muscle fibres after exercise of varying intensity and at varying pedalling rates. J Physiol 1974; 241:45-57.
22. Grisdale RK, Jacobs I, Cafarelli E. Relative effects of glycogen depletion and previous exercise on muscle force and endurance capacity. J Appl Physiol 1990;69:1276-82.

23. Heubert RAP, Quaresima V, Laffite LP, Koralsztein JP, Billat VL. Acute moderate hypoxia affects the oxygen desaturation and the performance but not the oxygen uptake response. Int J Sports Med 2005;26:542-51.

24. Pichon AP, de Bisschop C, Roulaud M, Denjean A, Papelier Y. Spectral analysis of heart rate variability during exercise in trained subjects. Med Sci Sports Exerc 2004; 36:1702-08.

25. Cottin F, Medigue C, Leprêtre PM, Papelier Y, Koralsztein JP, Billat V. Heart rate variability during exercise performed below and above ventilatory threshold. Med Sci Sports Exerc 2004;36:594-600.

26. Tulppo MP, Mäkikallio TH, Seppänen T, Laukkanen RT, Huikuri HV. Vagal modulation of heart rate during exercise: effects of age and physical fitness. Am J Physiol (Heart Circ. Physiol.) 1998; 274(2):H424-H9.

27. Fronchetti L, Nakamura FY, De-Oliveira FR, Lima-Silva AE, Lima JRP. Effects of high-intensity interval training on heart rate variability during exercise. JEPonline 2007; 10(4):1-9.

28. Araújo CGS. Fast "ON" and "OFF" heart rate transients at different bicycle exercise levels. Int J Sports Med 1985;6(2):68-73.

29. Mizuo J, Nakatsu T, Murakami T, Kusachi S, Tominaga Y, Mashima K, Uesugi T, Ueda H, Suezawa C, Tsuji T. Exponential hyperbolic sine function fitting of heart rate response to constant load exercise. Jpn J Physiol 2000;50(4):405-12.

30. Lima-Silva AE, De-Oliveira FR, Nakamura FY, Gevaerd MS. Effect of carbohydrate availability on time to exhaustion in exercise performed at two different intensities. Braz J Med Biol Res 2009;42(5):404-412.

\section{Endereço para correspondência}

Adriano Eduardo Lima da Silva

Faculdade de Nutrição

Universidade Federal de Alagoas (UFAL)

Avenida Lorival Melo Mota, S/N

Campus A.C. Simões

Tabuleiro do Martins

CEP: 57072-970 - Maceió, AL. Brasil

E-mail: adrianosilva@usp.br 